\title{
Utilização de partículas de ZnO:Mn para a degradação do azul de metileno por processo de fotocatálise
}

\section{(Use of ZnO:Mn particles for degradation of methylene blue by photocatalysis process)}

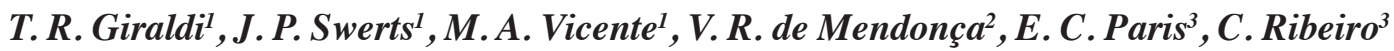 \\ ${ }^{1}$ Instituto de Ciência e Tecnologia, UNIFAL-MG, Rod. José Aurélio Vilela, 11999, Poços de Caldas, \\ $M G$ 37715-400 \\ ${ }^{2}$ Instituto Federal de Educação, Ciência e Tecnologia de S. Paulo, Campus Itapetininga, \\ Av. João Olímpio de Oliveira, 1561, Itapetininga, SP 18208-000 \\ ${ }^{3}$ Embrapa Instrumentação, R. XV de Novembro, 1452, S. Carlos, SP 13560-970 \\ tania.giraldi@unifal-mg.edu.br, jefersonps21@hotmail.com,maquelyaspr@oi.com.br,vrm@ifsp.edu.br, \\ elaine.paris@embrapa.br,caue.ribeiro@embrapa.br
}

\begin{abstract}
Resumo
A gestão do uso da água no ambiente agrícola é fator decisivo para a construção de métodos produtivos ambientalmente sustentáveis. A fotocatálise é um método promissor para a descontaminação de águas. Este artigo teve como proposta avaliar o potencial fotocatalítico de partículas de $\mathrm{ZnO}$ puro e dopado com manganês. Estes materiais foram produzidos pelo método dos precursores poliméricos e caracterizados por difratometria de raios $\mathrm{X}$, adsorção de $\mathrm{N}_{2}$ em baixa temperatura, espectroscopia na região do infravermelho e do ultravioleta e fotoluminescência. Para avaliação da eficiência dos fotocatalisadores obtidos, ensaios de fotodegradação foram realizados utilizando-se o corante azul de metileno sob radiação UVC. As partículas apresentaram-se monofásicas, porém com baixa área superficial específica. A amostra de $\mathrm{ZnO}$ com teor de dopagem de $0,25 \%$ de $\mathrm{Mn}$ apresentou maior eficiência no processo fotocatalítico para a degradação do corante azul de metileno. Esta eficiência foi relacionada com o maior valor de energia de band gap e menor taxa de recombinação eletrônica, o que permite maior formação de radicais de hidroxila, compostos estes responsáveis pela degradação do corante.
\end{abstract}

Palavras-chave: $\mathrm{ZnO}$, partículas, fotocatalisadores.

\begin{abstract}
The management of water use in the agricultural environment is a key factor to build new environmentally sustainable productive methods. Photocatalysis is a promising method for water decontamination. This research paper aimed to evaluate the photocatalytic potential of pure and $\mathrm{Mn}$-doped $\mathrm{ZnO}$ particles. The materials were obtained by the polymeric precursor method, and characterized by X-ray diffraction, $\mathrm{N}_{2}$ adsorption in low temperature, infrared and ultraviolet spectroscopy, and photoluminescence. In order to evaluate ZnO:Mn particles photoefficiency, experiments were carried out by applying the methylene blue dye solution to photodegradation under UVC exposure. The particles had ZnO single-phase, but low specific surface area. The sample ZnO:0.25\% Mn presented higher efficiency in the methylene blue photocatalytic degradation test. This efficiency was related to a higher band gap energy value and a lower rate of electron recombination, which allows greater formation of hydroxyl radicals, which are responsible for dye degradation.
\end{abstract}

Keywords: $\mathrm{ZnO}$, particles, photocatalyts.

\section{INTRODUÇÃO}

A gestão do uso da água é hoje um dos principais desafios para um desenvolvimento sustentável, principalmente em nações que dependem economicamente da produção agrícola, como é o caso do Brasil, detentor de cerca de $12 \%$ das reservas de água doce do planeta. Das fontes de água para a agricultura, $44 \%$ da oferta anual são advindas da precipitação, e 56\% correspondem à água do solo. Essa última parcela é extremamente sensível à contaminação, e impõe a necessidade da utilização racional do recurso, face à grande demanda atual [1]. Processos de descontaminação são, desta forma, necessários tanto na recuperação de aquíferos contaminados como na prevenção de contaminações futuras pelo adequado tratamento dos efluentes antes do descarte. Dentre os vetores de contaminação da água no meio agrícola, merecem particular destaque os pesticidas e fertilizantes químicos (agroquímicos). O uso indiscriminado de agroquímicos, associado à irrigação sem controle, tem comprometido as reservas hídricas de regiões no país, especialmente aquelas nas quais houve intensa expansão de atividades específicas, como a soja, nas fronteiras amazônicas 
com o Mato Grosso [1]. Atualmente, estes processos são considerados a segunda maior fonte de contaminação da água potável nos países em desenvolvimento [2].

No meio ambiente, estudos acerca da contaminação associada a agroquímicos demonstram efeitos drásticos, como aumento de anormalidades sexuais em anfíbios [3]. Em humanos, agroquímicos raramente são associados diretamente a doenças ou distúrbios de saúde, porém sabe-se que apresentam um efeito cumulativo no organismo, sendo que alguns compostos já são diretamente associados à maior incidência de distúrbios, como o câncer de mama [4]. Demais consequências da exposição humana por longos períodos de tempo a estes compostos ainda são pouco conhecidos [4]. O tratamento destes contaminantes em geral é complexo. Em muitos casos só se obtém a eficiência desejada para o tratamento adequado através da combinação de dois ou mais processos. Entre os mais importantes, os processos oxidativos avançados envolvem a geração e consumo de uma espécie altamente oxidante e não seletiva, destacandose principalmente o radical hidroxila $\left({ }^{\circ} \mathrm{OH}\right)$ e, em alguns casos, o oxigênio atômico [O(1D)] [5].

Uma maneira de se gerar o radical hidroxila é por meios fotoquímicos com radiação ultravioleta (UV) em combinação com $\mathrm{O}_{3}, \mathrm{H}_{2} \mathrm{O}_{2}$ [6], pelo processo Fenton [7], e ainda através de processos fotocatalíticos na faixa de luz ultravioleta - visível (por exemplo, luz solar) sobre a superfície de semicondutores, processo denominado fotocatálise heterogênea $[8,9]$. Os fotocatalisadores apresentam diversas aplicações. No entanto, nos últimos 20 anos os processos oxidativos avançados intermediados por catalisadores heterogêneos têm chamado atenção. A fotocatálise heterogênea é um método bastante versátil e promissor, pois promove a degradação de poluentes, apresenta custo relativamente baixo e possui alta eficiência mesmo para poluentes resistentes [10]. Normalmente, semicondutores, tais como $\mathrm{TiO}_{2}$ e $\mathrm{ZnO}$, dentre outros, são utilizados como fotocatalisadores. Isto se deve às estruturas eletrônicas destes materiais, caracterizadas por uma banda de valência preenchida e uma banda de condução vazia quando estes estão no estado fundamental [11].

Uma ferramenta importante para variações no processo é promover a alteração da estrutura física e eletrônica dos semicondutores por meio de dopagem. Esta estratégia permite a incorporação de novos elementos na estrutura reticular, alterando o posicionamento das bandas de condução e valência, promovendo alteração no valor da energia de band gap [12]. No caso da dopagem com íons metálicos (metais de transição, como $\mathrm{V}, \mathrm{Mn}, \mathrm{Cu}, \mathrm{Co}$ e $\mathrm{Ni}$ ), este valor de band gap pode ser reduzido [13]. Além disso, a dopagem também possibilita que o semicondutor tenha sua absorção estendida de comprimentos de onda da região do UV para a região do visível. Tomando como base o mecanismo de fotoatividade dos semicondutores, a dopagem dos mesmos favorecendo a fotoativação pela luz visível pode ser explicada pelo surgimento de novos níveis de energia produzidos entre as bandas de valência e condução do material (Fig. 1). Estes níveis de energia são gerados pela dispersão das partículas do dopante na matriz do semicondutor e o elétron pode ser excitado da banda de valência (BV) para a banda de condução (BC) por energias menores, promovendo a formação de um par de cargas que migram para a superfície da partícula desencadeando reações. Neste caso há a possibilidade de um benefício adicional causado pela presença do dopante. A dopagem pode impedir que haja recombinação do par elétron-buraco $\left(\mathrm{e}^{\prime} / \mathrm{h}^{\mathrm{n}}\right)$ durante a irradiação, acarretando em um aumento da fotoatividade do material [10].

$\mathrm{O}$ efeito da dopagem nas propriedades ópticas do $\mathrm{ZnO}$ pode ser observado pelo trabalho de Ullah e Dutta [14], que estudaram a atividade fotocatalítica do $\mathrm{ZnO}$ dopado com manganês frente à degradação do corante azul de metileno usando como fonte de radiação luz UV e visível. Para a síntese das nanopartículas, os autores usaram soluções etanólicas de acetato de zinco, hidróxido de sódio e acetato de manganês, todas a $50^{\circ} \mathrm{C}$. Após a mistura das soluções de acetato de zinco e manganês, adicionou-se solução de hidróxido de sódio, ocorrendo a precipitação a $65^{\circ} \mathrm{C}$. Opticamente, observou-se que houve um aumento na intensidade de absorção na região do azul para as partículas dopadas, sugerindo que o sistema ZnO:Mn absorve mais luz visível, podendo ser usado como um eficiente fotocatalisador quando irradiado por este tipo de radiação. Entretanto, os autores assumiram que em altas concentrações o dopante pode reagir mais com o oxigênio e formar $\mathrm{MnO}_{x}$ ao invés de ocorrer substituição intersticial no cristal de $\mathrm{ZnO}$, anulando o efeito de deslocamento da energia de band gap [14].

No presente trabalho, o sistema $\mathrm{ZnO}: \mathrm{Mn}$ foi avaliado frente à fotodegradação do corante azul de metileno. As partículas foram obtidas pelo método dos precursores poliméricos. Este método consiste na formação de uma cadeia polimérica, com os metais complexados em sítios ativos. Esta cadeia é formada, primeiramente, na reação do metal

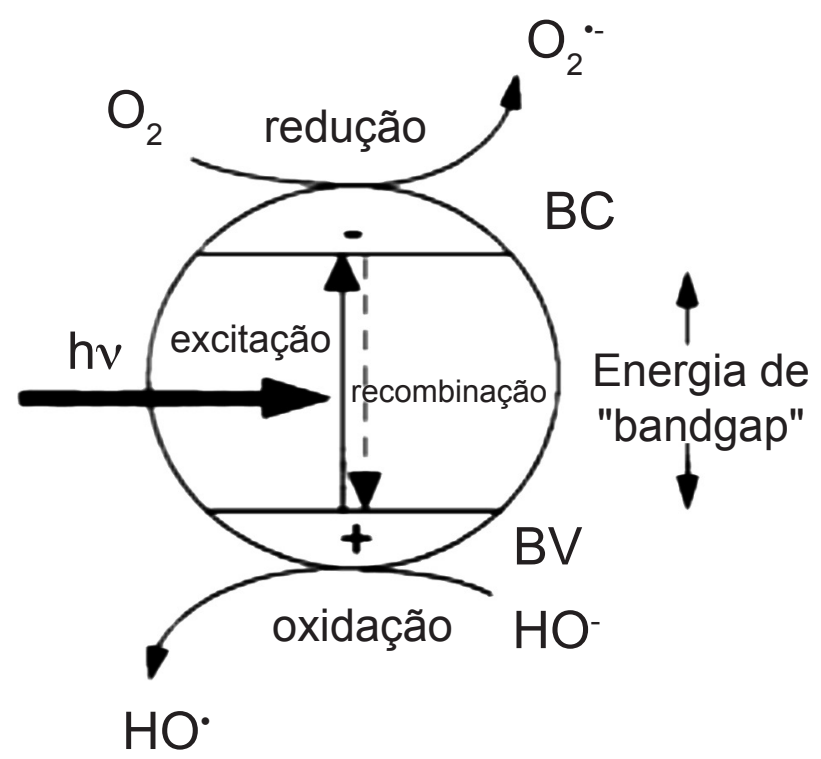

Figura1: Mecanismo de fotocatálise de um semicondutor [11]. [Figure 1: Photocatalysis mechanism of a semiconductor [11].] 
com um agente complexante, geralmente o ácido cítrico. Ao passo que os metais estejam complexados nos sítios ativos do ácido cítrico, forma-se então o citrato metálico. Por fim, as moléculas de citrato são polimerizadas com a adição do agente polimerizante, usualmente etilenoglicol [15]. Este método é bastante utilizado e tem como vantagens a síntese em baixas temperaturas e a baixa contaminação. Os objetivos deste trabalho foram a obtenção de partículas de $\mathrm{ZnO}$ dopado com manganês e avaliação de seu potencial fotocatalítico frente à fotodegradação do corante azul de metileno, molécula modelo para poluentes em água.

\section{METODOLOGIA}

Foram sintetizadas partículas de $\mathrm{ZnO}$ puro e dopado com manganês ( $\mathrm{ZnO}: \mathrm{Mn}$ ), nas proporções molares de 0,25 , 0,5 e $1,0 \% \mathrm{Mn}$. Para tanto, misturou-se acetato de zinco di-hidratado (Ecibra) com ácido cítrico (Alfa Aesar) na proporção molar 1:3. Essa mistura sólida foi adicionada aos poucos a uma solução previamente preparada de etilenoglicol (Ecibra), com álcool etílico absoluto (Ecibra). Nesta solução, a quantidade de etilenoglicol foi correspondente à proporção em massa de 3:2 em relação ao ácido cítrico. Para obtenção do material dopado, foi adicionado acetato de manganês tetra-hidratado (Alfa Aesar) à mistura sólida nas proporções molares citadas anteriormente. A mistura foi agitada a $70^{\circ} \mathrm{C}$ em pH controlado para a obtenção da solução polimérica. Para a obtenção das partículas e eliminação de material orgânico, as soluções poliméricas foram evaporadas e, em seguida, submetidas a tratamento térmico a $400{ }^{\circ} \mathrm{C}$ por $4 \mathrm{~h}$ com taxa de aquecimento de $20{ }^{\circ} \mathrm{C} / \mathrm{min}$. Os sólidos obtidos foram macerados e submetidos a um novo tratamento térmico para a cristalização do material. Para tal, utilizouse a temperatura de cristalização de $600{ }^{\circ} \mathrm{C}$, com taxa de aquecimento de $10^{\circ} \mathrm{C} / \mathrm{min}$.

A cristalinidade e análise das fases existentes nas partículas de $\mathrm{ZnO}$ puro e dopados com $\mathrm{Mn}$ foram avaliadas utilizando-se a técnica de difração de raios $\mathrm{X}$, DRX (Shimadzu, XRD-6000, $30 \mathrm{kV}$ e $30 \mathrm{~mA}$ ). A área superficial específica foi determinada utilizando-se a modelagem BET sobre os dados de adsorção de $\mathrm{N}_{2}$ a 77 K, em equipamento Micromeritics, ASAP 2000. Para a verificação de possíveis espécies adsorvidas na superfície das partículas, foram realizadas medidas de espectroscopia na região do infravermelho em espectrofotômetro Bruker I, FS66, utilizando-se pastilhas de KBr. Para determinação do valor de energia de band gap das partículas, foi utilizada espectroscopia na região do UV-visível no modo reflectância difusa. Por fim, espectroscopia de fotoluminescência foi realizada para o estudo de cargas fotogeradas nas partículas. Os espectros foram obtidos a partir de suspensão das amostras em água em um espectrômetro de luminescência Perkin Elmer, LS-50B.

A degradação do corante azul de metileno (AM) foi analisada para estudos sobre as propriedades fotocatalíticas das amostras. Assim, uma solução aquosa de AM $5 \mathrm{mg} / \mathrm{L}$ foi preparada. Em diferentes béqueres foram adicionados
$50 \mathrm{~mL}$ dessa solução e $0,01 \mathrm{~g}$ de fotocatalisador. O sistema foi mantido à temperatura constante próxima a do ambiente utilizando-se banho termostático, sob constante agitação e iluminado por quatro lâmpadas UVC (Philips TUV, 15 W, emissão máxima de $254 \mathrm{~nm}$ ). A degradação do corante foi realizada em triplicata e monitorada por medidas de absorbância, realizadas em um espectrômetro UV-Vis (Shimadzu, UV-1601PC), em diferentes tempos de exposição à luz da solução de corante contendo fotocatalisador.

\section{RESULTADOS E DISCUSSÃO}

A Fig. 2 ilustra os difratogramas de raios $\mathrm{X}$ das partículas de $\mathrm{ZnO}$ puro e dopado com $\mathrm{Mn}$ em diferentes concentrações. Observam-se picos referentes à fase $\mathrm{ZnO}$, de acordo com o arquivo JCPDS 36-1451. Não foram verificados picos referentes a fases relacionadas ao manganês. Este é um indicativo da dopagem de $\mathrm{Mn}$ na estrutura cristalina do $\mathrm{ZnO}$, conforme o esperado.

A Tabela I apresenta os dados de área superficial específica das amostras obtidas. Observa-se que as amostras apresentaram baixa área superficial específica. A amostra de $\mathrm{ZnO}$ dopado com $0,5 \%$ de manganês apresentou área superficial maior do que das outras amostras. A baixa área superficial é um fator que pode influenciar negativamente na eficiência fotocatalítica das partículas, uma vez que as reações ocorrem na superfície das mesmas [16].

Uma possível explicação para a baixa área superficial pode estar relacionada com a aglomeração das partículas no processo de tratamento térmico. Este fenômeno pode ser evidenciado por análises de espectroscopia na região do infravermelho, de acordo com a Fig. 3. Observa-se que, exceto na amostra de $\mathrm{ZnO}$, as partículas se encontram livres de íons adsorvidos em sua superfície. Isto faz com que ocorra contato entre as partículas e que, durante o tratamento térmico, possivelmente ocorra formação de pescoços com

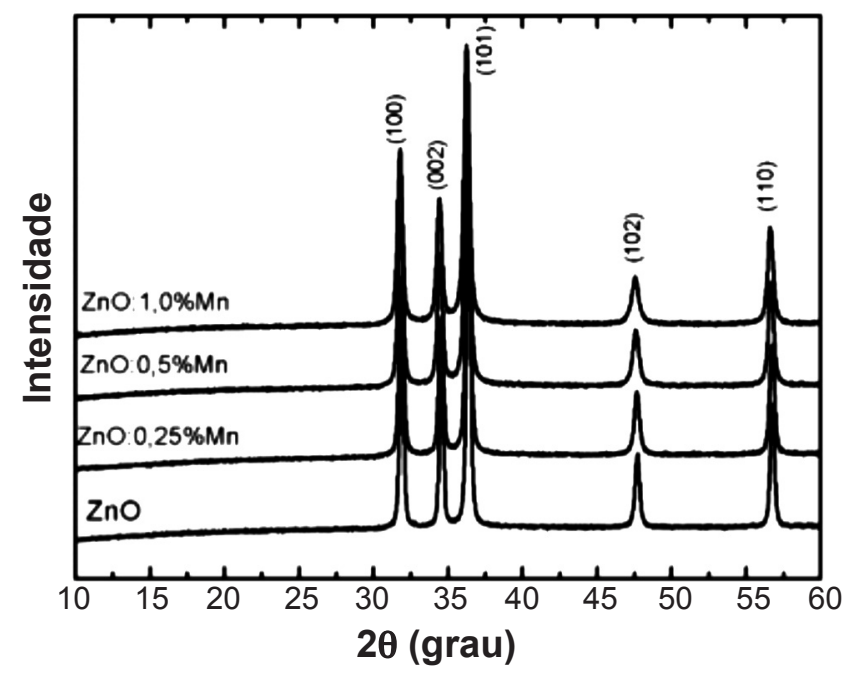

Figura 2: Difratogramas de raios $\mathrm{X}$ das partículas de $\mathrm{ZnO}$ puro e dopado com manganês.

[Figure 2: XRD patterns of pure and manganese-doped $\mathrm{ZnO}$ particles.] 
Tabela I - Área superficial das amostras de $\mathrm{ZnO}$ e ZnO:Mn. [Table I - Surface area of $\mathrm{ZnO}$ and $\mathrm{ZnO} \mathrm{OMn}$ samples.]

\begin{tabular}{cc}
\hline Amostra & Área superficial $\left(\mathrm{m}^{2} / \mathrm{g}\right)$ \\
\hline $\mathrm{ZnO}$ & 0,3125 \\
$\mathrm{ZnO}: 0,25 \% \mathrm{Mn}$ & 0,2473 \\
$\mathrm{ZnO}: 0,5 \% \mathrm{Mn}$ & 2,3644 \\
$\mathrm{ZnO}: 1,0 \% \mathrm{Mn}$ & 0,9785 \\
\hline
\end{tabular}

consequente aglomeração e posterior sinterização. Este fator gera diminuição da área superficial, conforme verificado por resultados anteriormente apresentados. Giraldi et al. [17] verificaram este fenômeno ao sintetizarem $\mathrm{ZnO}$ em diferentes temperaturas de tratamento térmico. $\mathrm{Na}$ amostra de $\mathrm{ZnO}$ verificou-se na faixa de $1600 \mathrm{~cm}^{-1}$ a presença de uma suave banda referente ao estiramento simétrico a assimétrico do ânion carboxilato [18]. Isto indica que há um resíduo de grupos de acetato na superfície das partículas. No entanto, acredita-se que a quantidade deste resíduo não seja suficiente para evitar a aglomeração das partículas.

Assumindo que o material é um semicondutor com band gap indireto, um gráfico da função modificada de KubelkaMunk R/S em função da energia luminosa [19] (Fig. 4) apresentou-se útil para a determinação do valor de energia de band gap dos materiais sintetizados. A energia de band gap das amostras foi determinada pelo prolongamento da curva de absorção apresentado na Fig. 4. A energia de band gap apresenta um aumento de $0,05 \mathrm{eV}$ para amostra dopada com $1 \%$ de $\mathrm{Mn}$, com relação ao $\mathrm{ZnO}$ puro, com decréscimo conforme diminuição da dopagem, chegando até um valor de 3,09 eV para amostra dopada com 0,25\% de Mn. Observase que o $\mathrm{ZnO}$ puro apresentou band gap intermediário em relação às amostras dopadas: $\mathrm{ZnO}: 0,25 \%$ apresentou menor band gap em relação ao $\mathrm{ZnO}$ puro, enquanto $\mathrm{ZnO} 0,0,5 \%$ e $\mathrm{ZnO}: 1,0 \%$ apresentaram maior band gap em relação ao $\mathrm{ZnO}$

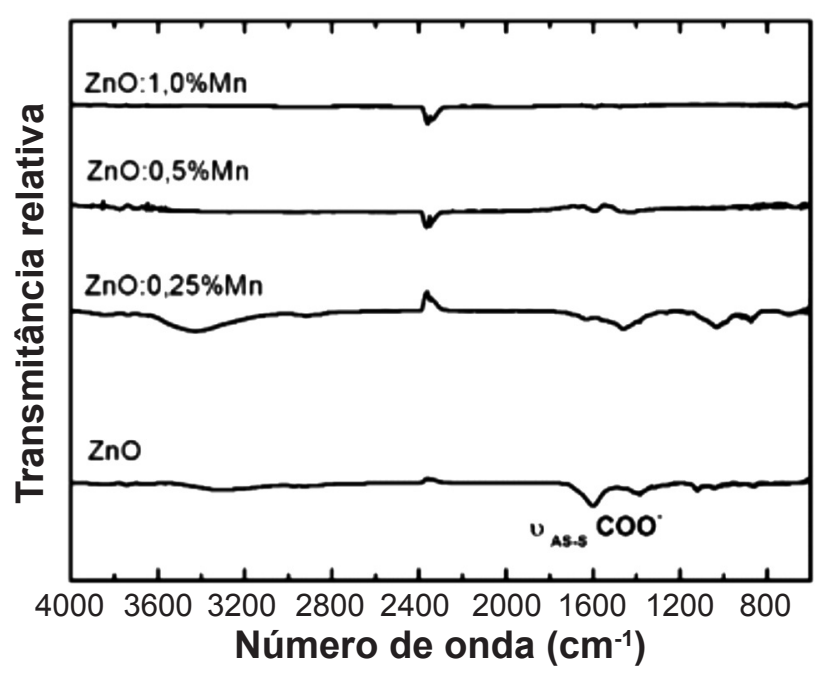

Figura 3: Espectroscopia na região do infravermelho das partículas de $\mathrm{ZnO}$ puro e dopado com manganês.

[Figure 3: Infrared spectroscopy of pure and manganese-doped $\mathrm{ZnO}$ particles.]

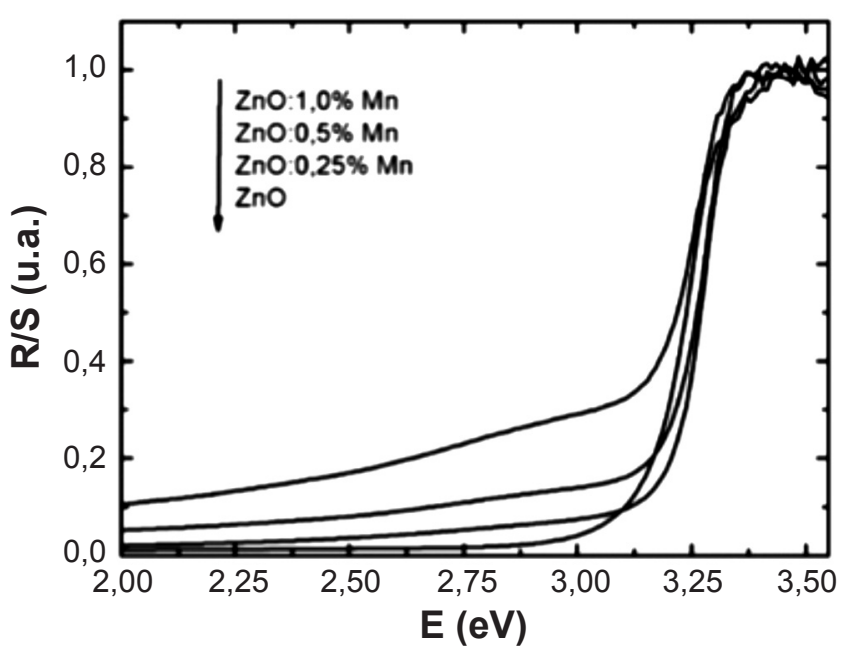

Figura 4: Espectros de reflectância difusa UV-Vis (função modificada de Kubelka-Munk R/S) em função da energia para partículas de $\mathrm{ZnO}$ puro e dopado com $\mathrm{Mn}$.

[Figure 4: Diffuse reflectance UV-Vis spectra (modified R/S Kubelka-Munk function) vs. energy for pure and manganese-doped $\mathrm{ZnO}$ particles.]

puro. A alteração do band gap em relação ao $\mathrm{ZnO}$ puro $(3,13$ $\mathrm{eV}$ ) era esperada e é um indício da efetividade do método de síntese na dopagem do $\mathrm{ZnO}$.

A espectroscopia de fotoluminescência (FL) é uma técnica que permite a determinação de defeitos e impurezas em semicondutores [20] e baseia-se na emissão radiativa espontânea dos materiais [21]. Neste trabalho, espectros de FL foram obtidos para uma melhor compreensão do comportamento fotocatalítico, uma vez que fotoefeitos são dependentes das características eletrônicas das partículas. Estes espectros são apresentados na Fig. 5, e todas as intensidades foram normalizadas para a amostra mais intensa. As intensidades dos espectros de FL podem ser entendidas como inversamente proporcionais à presença de centros de recombinação nas amostras [12]. Como pode ser verificada pela Fig. 5, ZnO puro apresentou maior intensidade do que as demais amostras. Possivelmente esta amostra proporciona uma menor recombinação não radiativa de cargas. Ainda, verifica-se que com o aumento da concentração de manganês ocorre a diminuição da intensidade dos picos, indicando a presença de centros de recombinação das cargas fotogeradas na estrutura cristalina do material [12].

Uma vez realizadas as caracterizações das partículas, as mesmas foram utilizadas para ensaios fotocatalíticos na degradação do corante azul de metileno (AM). A Fig. 6 ilustra resultados da fotodegradação, na região UVC, do corante na presença dos diferentes fotocatalisadores sintetizados. A diminuição da concentração do corante $(\mathrm{C} / \mathrm{Co})$ indica que o AM foi oxidado. De acordo com a Fig. 6, é possível verificar que a presença dos catalisadores acelera o processo de degradação do AM sob luz UVC, já que a solução de AM (branco) não apresentou alteração considerável de concentração. Além disso, constata-se que a amostra $\mathrm{ZnO} 0,0,25 \% \mathrm{Mn}$ apresenta a maior fotoatividade, uma vez que promoveu degradação de $78 \%$ do corante em $120 \mathrm{~min}$ 


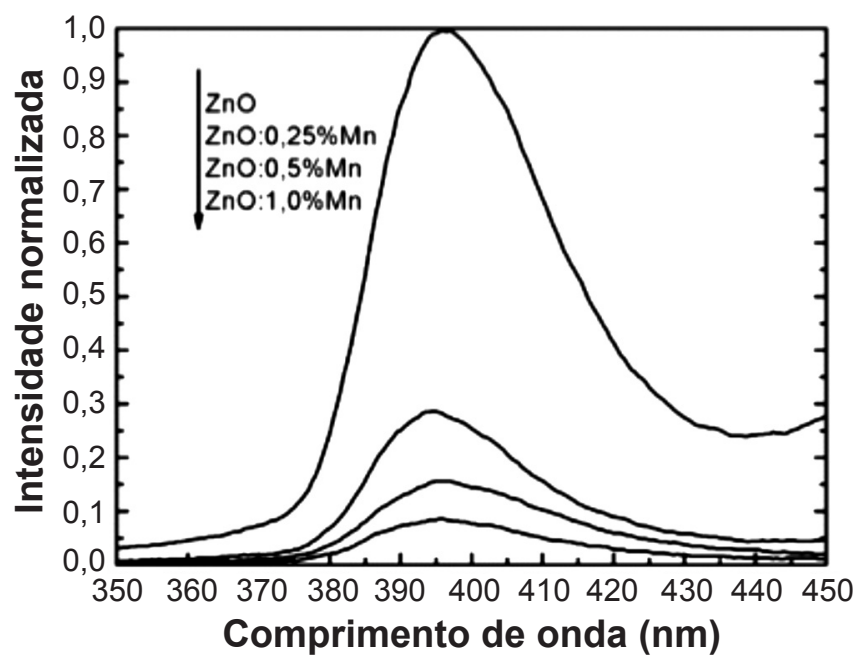

Figura 5: Espectros de fotoluminescência das partículas de $\mathrm{ZnO}$ puro e dopado com manganês.

[Figure 5: Photoluminescence spectra of pure and manganesedoped $\mathrm{ZnO}$ particles.]

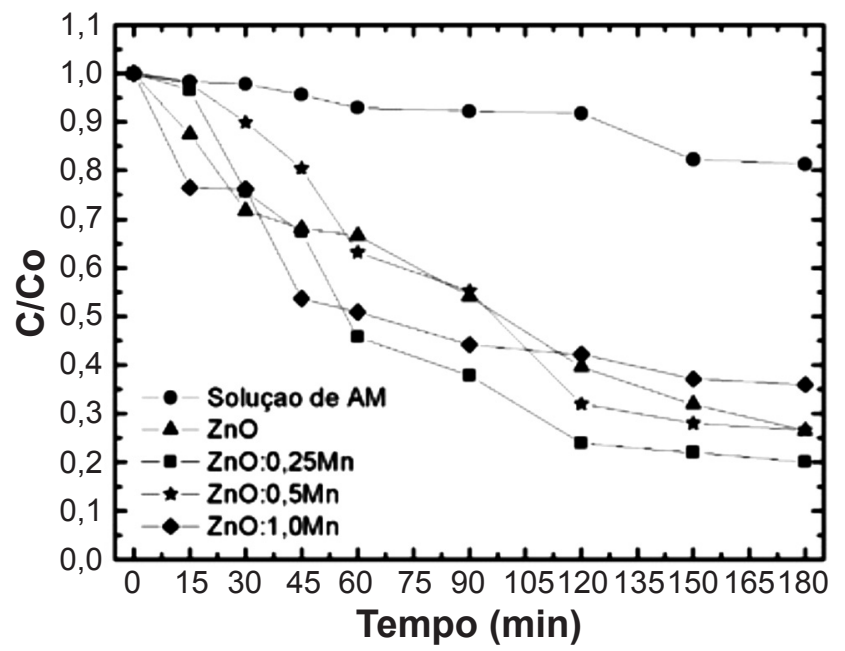

Figura 6: Resultados de ensaios fotocatalíticos na região UVC com partículas de $\mathrm{ZnO}$ puro e dopado com manganês.

[Figure 6: Results of photocatalytic tests in the UVC region with pure and manganese-doped $\mathrm{ZnO}$ particles.]

de exposição à radiação. Também se pode dizer que as amostras $\mathrm{ZnO}$ puro e $\mathrm{ZnO}: 0,5 \% \mathrm{Mn}$ apresentaram atividades semelhantes e, por fim, $\mathrm{ZnO}: 1,0 \% \mathrm{Mn}$ apresentou menor atividade fotocatalítica, degradando $60 \%$ do corante em 150 min. Estes resultados não podem ser atribuídos à área superficial das partículas, pois, de acordo com a Tabela I, as amostras apresentaram baixos valores de área superficial específica. No entanto, estes resultados podem ser atribuídos às propriedades eletrônicas dos materiais. A Tabela II ilustra a relação entre os resultados de fotocatálise e propriedades eletrônicas das partículas.

Observa-se que o material com maior band gap, $\mathrm{ZnO}: 0,25 \% \mathrm{Mn}$, apresentou também maior atividade fotocatalítica. Exceto para a amostra de $\mathrm{ZnO}$ puro, $\mathrm{ZnO}: 0,25 \% \mathrm{Mn}$ apresentou pico de fotoluminescência com maior intensidade em relação às outras amostras dopadas.
Tabela II - Relação entre resultados fotocatalíticos e propriedades eletrônicas das amostras de $\mathrm{ZnO}$ e $\mathrm{ZnO}: \mathrm{Mn}$. [Table II - Relationship between photocatalytic results and electronic properties of $\mathrm{ZnO}$ and $\mathrm{ZnO}: \mathrm{Mn}$ samples.]

\begin{tabular}{cccc}
\hline Amostra & $\begin{array}{c}\text { \% de } \\
\text { descoloração } \\
\text { em } 150 \text { min }\end{array}$ & $\begin{array}{c}\text { Band gap } \\
(\mathrm{eV})\end{array}$ & $\begin{array}{c}\text { Intensidade do } \\
\text { pico FL }\end{array}$ \\
\hline $\mathrm{ZnO}$ & 69 & 3,13 & 1,00 \\
$\mathrm{ZnO}: 0,25 \mathrm{Mn}$ & 78 & 3,18 & 0,28 \\
$\mathrm{ZnO}: 0,5 \mathrm{Mn}$ & 72 & 3,15 & 0,16 \\
$\mathrm{ZnO}: 1,0 \mathrm{Mn}$ & 62 & 3,09 & 0,08 \\
\hline
\end{tabular}

Isto indica, de forma discreta, ao comparar as amostras dopadas, que quanto maior o valor de energia de band gap e intensidade do pico FL, maior é a atividade fotocatalítica do material. Levando-se em consideração que a radiação UVC utilizada durante os ensaios de fotodegradação foi suficiente para excitação de todas as amostras, de acordo com os dados apresentados na Tabela II, esta tendência pode ser justificada pelo fato de que, com maior separação energética entre as bandas de valência e condução, o elétron presente na banda de condução deve apresentar menor taxa de recombinação eletrônica não radiativa. A não recombinação permite que as cargas fotogeradas atinjam a superfície do material, acarretando em uma maior taxa de formação dos radicais de hidroxila, promovendo, enfim, a fotodegradação do corante [16].

\section{CONCLUSÕES}

Este trabalho permitiu concluir que o método dos precursores poliméricos é eficaz na obtenção de partículas de $\mathrm{ZnO}$ dopadas com $\mathrm{Mn}$. As partículas apresentaram-se monofásicas, porém com baixa área superficial específica. A amostra de $\mathrm{ZnO}$ com teor de dopagem de 0,25\% Mn apresentou maior eficiência no processo fotocatalítico para a degradação do corante azul de metileno. Esta eficiência pode ser relacionada com o maior band gap e menor taxa de recombinação eletrônica, o que permite maior formação de radicais de hidroxila, compostos estes responsáveis pela degradação do corante.

\section{AGRADECIMENTOS}

Ao Laboratório Interdisciplinar de Eletroquímica e Cerâmica (LIEC), Fapemig (Projeto APQ-01316-12) e CNPq, pelo apoio concedido.

\section{REFERÊNCIAS}

[1] MMA/SRH, Caderno setorial de recursos hídricos: agropecuária, Ministério do Meio Ambiente (2006).

[2] A.D. Guardo, A. Finizio, Sci. Total. Environ. 545 (2016) 200.

[3] T. Hayes, K. Haston, M. Tsui, A. Hoang, C. Haefiele, A. Vonk, Nature 419 (2002) 895. 
[4] D. Pestana, D. Teixeira, A. Faria, V. Domingues, R. Monteiro, C. Calhau, Environ. Toxic. 30 (2015) 168.

[5] M.A. Henderson, Surf. Sci. Report. 66 (2011) 185.

[6] S. Chiron, A. Fernandez-Alba, A. Rodriguez, E. GarciaCalvo, Water Res. 34, 2 (2000) 366.

[7] F. Haber, J. Weiss, Proceedings of the Royal Society of London, Series A, Math. Phys. Sci. 147, 861 (1934) 1934.

[8] K.M. Lee, C.W. Lai, K.S. Ngai, J.C. Juan, Water Res. 88 (2016) 428.

[9] S.I. Malato, M.I. Maldonado, P. Fernández-Ibáñez, I. Oller, I. Polo, R. Sánchez-Moreno, Mater. Sci. Semicon. Proc. 42 (2016) 15.

[10] H.A.J.L. Mourão, V.R. de Mendonça, A.R. Malagutti, C. Ribeiro, Quim. Nova 32 (2009) 2181.

[11] V.R. de Mendonça, "Nanocristais de $\mathrm{TiO}_{2}$ : relação entre parâmetros morfológicos estruturais e fotocatálise", Diss. Mestrado, Centro de Ciências Exatas e de Tecnologia, Universidade Federal de S. Carlos, S. Carlos (2010).

[12] W.D. Callister Jr., Ciência e Engenharia de Materiais: uma Introdução, LTC, Rio de Janeiro (2002).

[13] A. Zaleska, Physicochem. Probl. Mi. Proc. 42 (2008)
211.

[14] R. Ullah, J. Dutta, J. Hazard. Mater. 156 (2008) 194.

[15] M. Arima, M. Kakihana, Y.J. Nakamura. J. Am. Ceram. Soc. 79 (1996) 11.

[16] V.R. de Mendonça, C.J. Dalmaschio, E.R. Leite, M. Niederberger, C. Ribeiro, J. Mater. Chem. A, 3 (2015) 2216. [17] T.R. Giraldi, G.V.F. Santos, V.R. Mendonça, C. Ribeiro, I.T. Weber. J. Nanosci. Nanotechnol. 11, 1 (2011) 3635.

[18] R.M. Silverstein, G.C. Bassler, T.C. Morrill, Spectrometric Identification of Organic Compounds, $5^{\text {th }} \mathrm{Ed}$., John Wiley and Sons, New York (1991).

[19] S. Sakthivel, M. Janczarek, H.J. Kisch, Phys. Chem. B, 108 (2004) 19384.

[20] T.S. Burger, "Desenvolvimento de filmes de $\mathrm{ZnO}$ para aplicação em fotocatálise", Diss. Mestrado, Instituto de Química, Universidade Federal do Rio Grande do Sul, Porto Alegre (2011).

[21] E. Laureto, I.F.L. Dias, J.L. Duarte, D.O. Toginho Filho, S.A. Lourenço, E.A. Meneses, Semina: Ciências Exatas e Tecnológicas, Londrina 26, 1 (2005) 23. (Rec. 23/10/2015, Rev.09/03/2016, Ac. 04/05/2016) 